Japan. J. Med. Sci. Biol., 20, 329-339, 1967

\title{
SUSCEPTIBILITY OF CELL CULTURES FROM VARIOUS MAMMALIAN TISSUES TO THE INFECTION BY SHIGELLA
}

\author{
HidEMASA OGAWA, HiROSHI YOSHIKURA, \\ AKIKO NAKAMURA* AND RINTARO NAKAYA* \\ Department of Pathology, and * Department of Bacteriology I, \\ National Institute of Health, Tokyo
}

(Received : March 11th, 1967)

SUMMARY : Comparison of cell-infection rates by Shigella was made among primary or secondary cell cultures and established cell lines isolated from various mammalian tissues.

Cell cultures originating from the guinea pig cornea and the mouse lung showed a high infection rate with virulent strains of Shigella flexneri 2a, 3a and $S$. dysenteriae 1 . The infection rate in the former cell cultures was lower after a long period of subculture, while in the latter it was high even after more than 100 subcultures performed over 3 years. Cell cultures of both human and monkey origins including primary or secondary cultures from the intestine showed a low infection rate with all types of Shigella.

By the cell culture procedures used in the present work, animal species- or organ-specificity as found in the natural susceptibility to Shigella infection did not show any consistent correlationship with the infection rate in the cell culture from corresponding animals or organs.

Compared with other types of Shigella S. sonnei clearly showed a low cell infectivity. This fact agrees with the pathogenic behavior of $S$. sonnei which causes generally mild symptoms in human bacillary dysentery, experimental Shigellosis in monkeys, and keratoconjunctivitis in guinea pigs.

No difference in cell-infection rate was observed between primary cultures (polygonal cells) of the guinea pig cornea, which acquired resistance in vivo after recovery from the local infection with a virulent strain of Shigella, and those of the untreated control cornea. Irrespective of whether or not the pretreatment was made, the presence of hyperimmune serum against the infecting bacilli in the cell culture medium resulted in a decrease in the infection rate of cornea cells. This is considered to be caused by agglutination of the bacilli in extracellular environment.

\section{INTRODUCTION}

LaBrec et al. (1964) and Ogawa et al. (1966a) pointed out penetration into epithelial cells of the intestinal mucous membrane and intracellular multiplication of Shigella organisms as the essential factors determining the pathogenicity. Watkins (1960), LaBrec

* Presented in part at Symposium on Experimental Shigellosis: Infectivity and Pathogenicity of Shigella, National Institute of Health, Tokyo, November, 1966.

小河 秀䜣・吉倉 広 (国立予防衛生研究所病理部)

中村 明子・中谷 林太郎（国立予防衛生研究所細菌第一部） 
et al. (1964) and Ogawa et al. (1967), furthermore, demonstrated that the ability of the bacilli to induce the disease in experimental animals agreed fairly well with their ability to infect cell cultures in vitro. This fact made it possible to investigate the pathogenicity of Shigella under simplified conditions of cell culture. However, infection rates in the cell lines used by them were not high enough for quantitative analysis on the pathogenicity of Shigella (Ogawa et al., 1967), and therefore it became necessary to find out some cell lines with a higher infection rate. In the present study, comparison of cellinfection rates with Shigella was made among primary cell cultures including those of young subcultures and established cell lines isolated from various mammalian tissues. In this connection, the relation between cell-infection rate and susceptibility of various animals to Shigella infection will be discussed.

Another problem in close relation to this subject was concerned with a remarkable increase in local resistance acquired in the eyes of the guinea pigs recovering from keratoconjunctivitis shigellosa, in which non-specific factor played an important role (Ogawa et al., 1966 b). Whether or not this increased local resistance could be reproduced in cultured cells in vitro was also examined.

\section{MATERIALS AND Methods}

Cell cultures: Cell cultures employed are listed in Table 1.

Media : Eagle's minimal essential medium supplemented with $10 \%$ inactivated calf serum was used for growing cells and also as an infection media (Ogawa et al., 1967). For growth of the Shigella organisms Penassay broth agar (Difco) was used (Ogawa et al., 1967).

Bacterial strains and infection procedures: Shigella flexneri 2a strain 5503 was isolated from the affected colon of a monkey (No. 6619) after oral inoculation with $S$. flexneri 2a strain 5503 (Honjo et al., 1964). The colon had been stored at $-20 \mathrm{C}$ for about 8 months. S. flexneri 3a strain 638-66 and 23-65, S. dysenteriae 1 strain 143365 and $S$. sonnei strain 18-65 were recent isolates from feces of patients and maintained in cooked meat media (Difco) at room temperature. Besides those strains, several strains of $S$. flexneri and $S$. sonnei which were isolated freshly from patients or carriers were employed in experiments which compared the cell-infectivity between two subgroups of Shigella.

Confluent cell monolayers obtained from various tissues or stock cultures were grown in a small square tube containing $2 \mathrm{ml}$ of infection medium. Each tube was added with $0.2 \mathrm{ml}$ of bacterial suspension containing $1 \times 10^{9}$ cells per $\mathrm{ml}$ and then incubated at $37 \mathrm{C}$ for $2 \mathrm{hr}$. After $2 \mathrm{hr}$ of infection period, the cell monolayers were washed twice with balanced salt solution and added fresh media to minimize extracellular bacilli. Enumeration of the infected cells was carried out microscopically using specimens stained with Giemsa solution $6 \mathrm{hr}$ after infection. Details of the infection procedures were described in a previous publication by Ogawa et al. (1967).

For cell culture studies on the acquired resistance to Shigella infection, primary cultures were prepared from the eye of guinea pigs locally immunized with $S$. flexneri 2a strain 5503 and also, as control, from the other eye. Immunization procedure was described in the previous report (Ogawa et al., 1966 b). Four cell cultures from the conjunctiva and cornea grown for 4 or 14 days were challenged with strain 5503 in the presence or absence of hyperimmune serum of guinea pigs against strain 5503 (it 
Table 1. Cell cultures employed

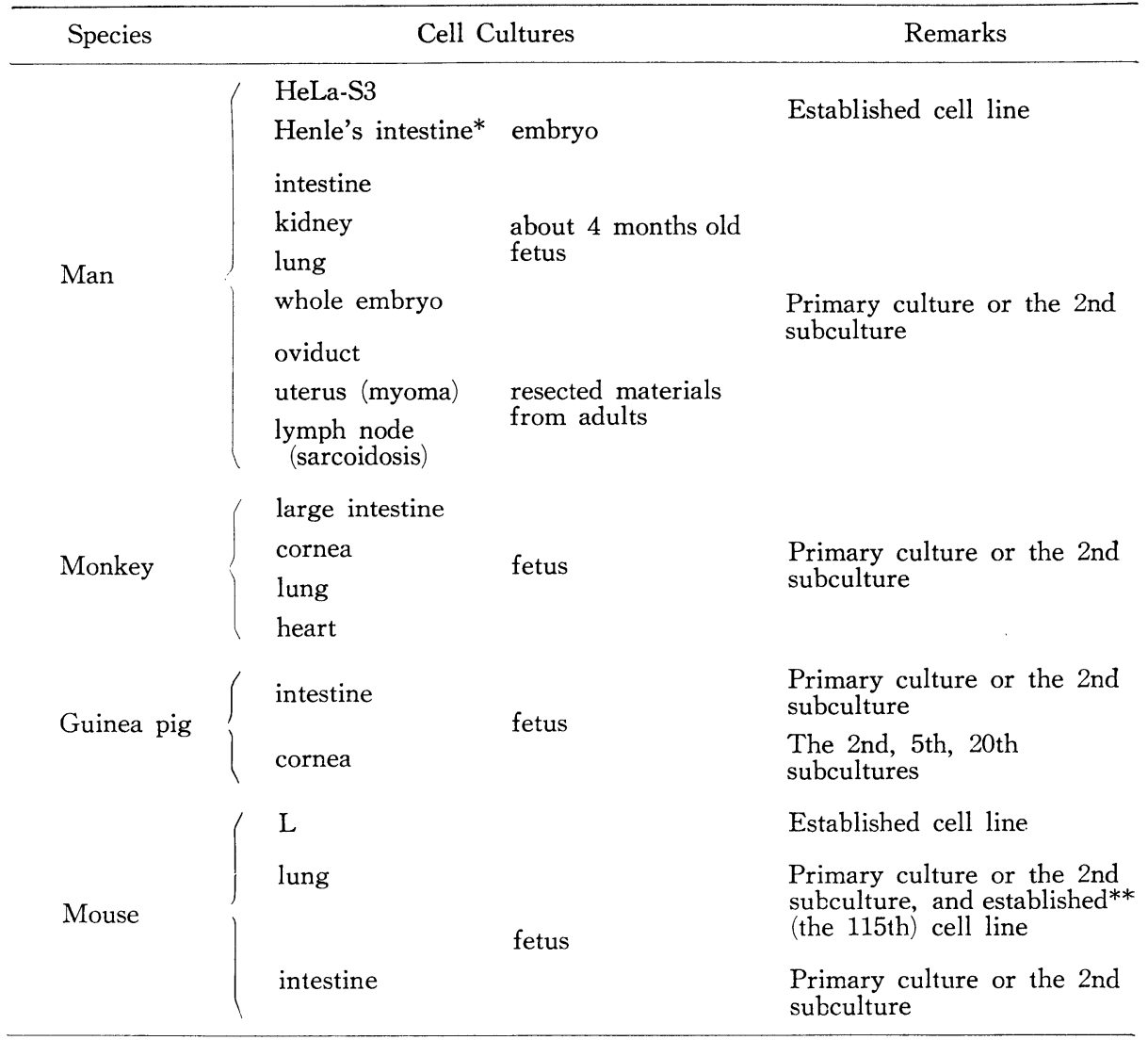

* Henle and Deinhardt (1957).

** Yoshikura, Hirokawa and Yamada (1966).

possessed an agglutinin titer of $1: 1,280$ and was added to a final dilution of $1: 10$; Ogawa et al., 1967).

\section{RESULTS}

\section{Comparison of Cell infection Rates with Shigella among Cell Cultures from Various Mammalian Tissues}

Fig. 1 presents cell infection rates with $S$. flexneri 2a strain 5503, S. flexneri 3a strains 638-66 and 23-65, and $S$. dysenteriae 1 strain 1433-65 diagramatically. Regarding the animal species, guinea pig and mouse cells showed in general a higher infection rate than human or monkey cells with each strain of Shigella tested. Among cell cultures from guinea pig tissues, cornea cells within the 5 th subculture showed an infection rate of about $10 \%$ with strains 5503 (Fig. 5), 638-66 and 1433-65, and more than $15 \%$ with strain 23-65, whereas the 20th subculture of cornea cells reduced the infection rate to approximately half that of the 5th subculture. In the case of cultures derived from 
A.

S. flexneri

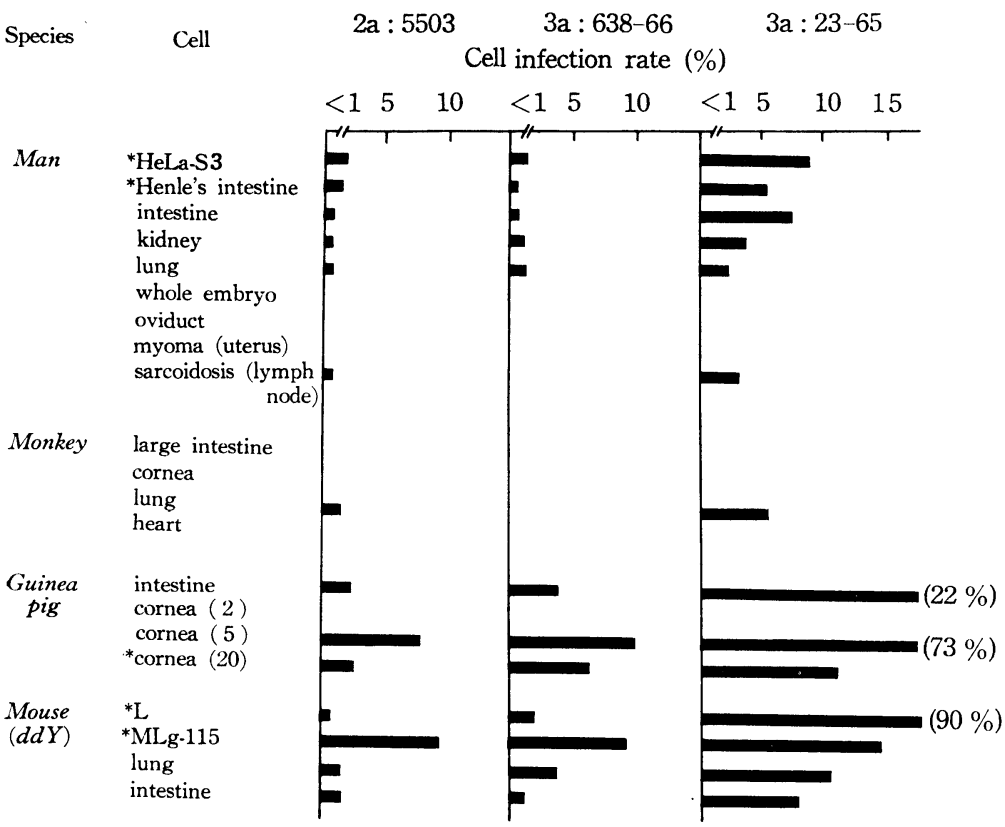

B.

S. dysenteriae 1, 1433-65

Exp. 1

Exp. 2

Exp. 3

Cell infection rate $(\%)$

Man

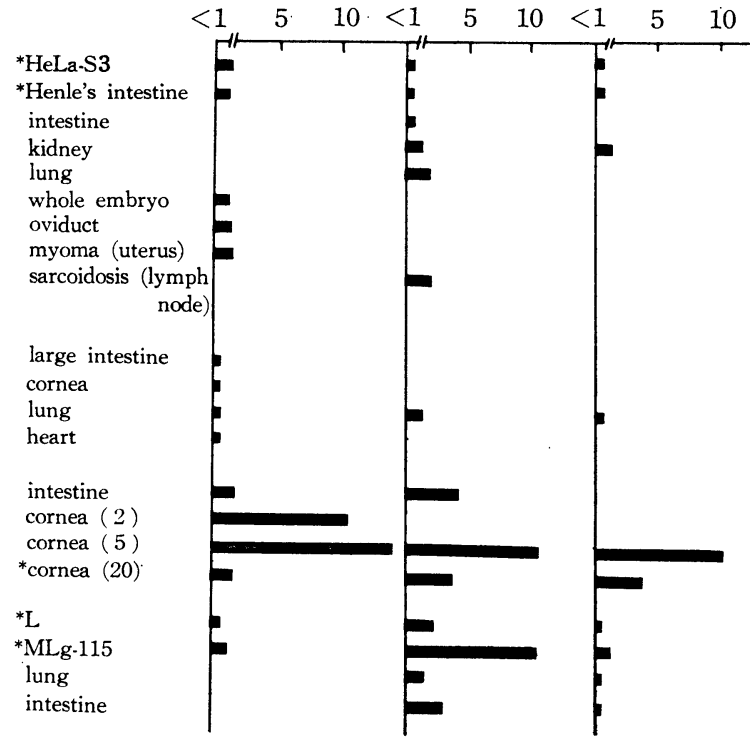

Fig. 1. Comparison of the rate of infection with Shigella among cell cultures from various mammalian tissues. Inoculum size : $2 \times 10^{8} /$ tube * Established cell line. 
mouse lung cells, the 115th subculture of an established cell line showed an infection rate of about $10 \%$ higher than that of primary culture of the lung with strains 5503 (Fig. 6) and 638-66, and this difference was about $15 \%$ with strain $23-65$. The infection rate of $\mathrm{L}$ cells, however, was as low as that of either human or monkey cells, except with $S$. Alexneri 3a strain 23-65. Cell cultures from the intestine both of guinea pigs and of mice were relatively low in the cell infection rate when compared with other primary cultures.

Because our experience indicated that actual cell infection rates varied from experiment to experiment even when a given bacterial strain and a given cell line were used, as can be seen in Fig. $1 \mathrm{~B}$, the reproducibility of results described above was tested in three independent experiments with $S$. dysenteriae 1 strain 1433-65. The results confirmed that the overall susceptibility pattern of cell cultures to infection by the bacilli did not show any significant difference among the experiments, though some minor variations of cell infection rates occurred.

\section{Comparison of Cell Infectivity between S. flexneri and S. sonnei}

It was difficult to compare the cell infectivity among the types of Shigella, since Cell infection rate $(\%)$

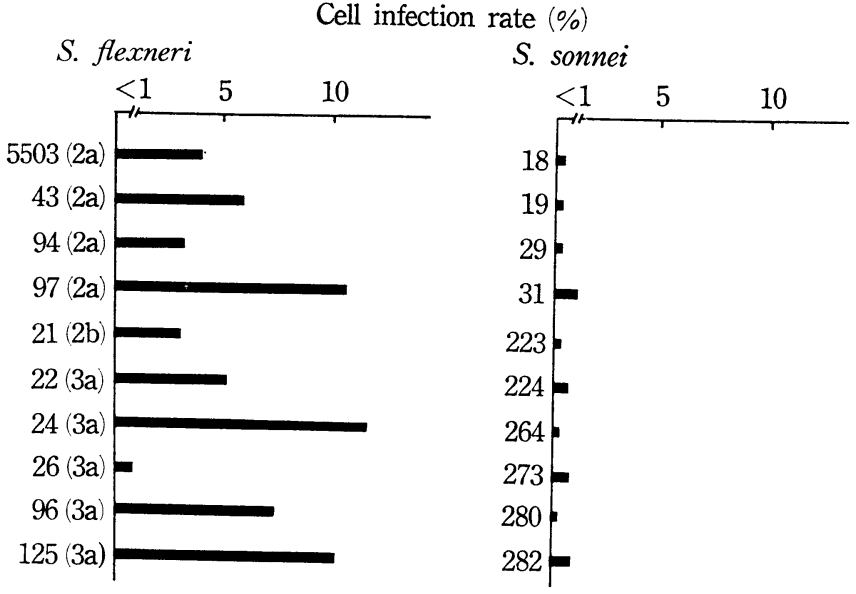

A. Infection rate of HeLa cells to $S$. flexneri and $S$. sonnei isolated newly from patients or carriers. Inoculum size : $5 \times 10^{8} /$ tube.

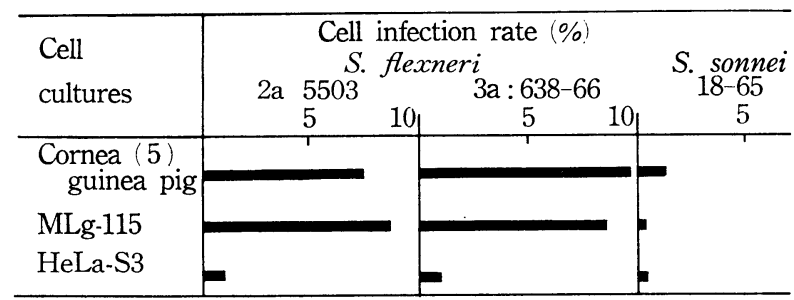

B. Infection rate of guinea pig cornea cells, mouse lung cells and HeLa cells to $S$. flexneri 2 a strain 5503 , and $S$. flexneri 3 a strain 638-66, and $S$. sonnei strain 18-65. Inoculum size : $2 \times 10^{8 / \text { tube. }}$

Fig. 2. Comparison of cell infectivity between S. flexneri and S. sonnei. 

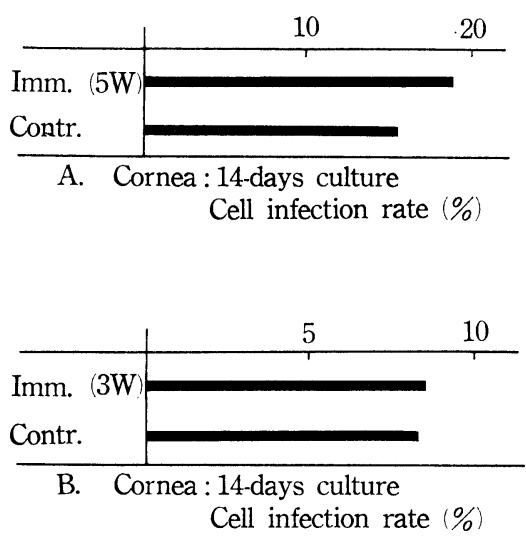

Fig. 3. Comparison of infection rates of cornea cells from locally immunized and control eyes challenged with Shigella flexneri 2a strain 5503.

actual cell infection rates varied according to the period after isolation and the media used for preservation of organisms tested. However, as it has been pointed out that $S$. sonnei causes milder clinical signs of dysentery not only in human beings but also in experimental animals, cell infection rates were compared between $S$. flexneri and $S$. sonnei which were newly isolated from dysentery patients and carriers. Fig. 2 shows cell infection rates of strains of $S$. flexneri and $S$. sonnei to HeLa-S3 cells (a resistant cell group), guinea pig cornea cells and mouse lung cells (a susceptible cell group). To any cell culture the infectivity of all strains of $S$. sonnei tested was definitely lower than that of $S$. flexneri.

\section{Comparison of Cell Infection Rates of Cornea and Conjunctiva Cells from Locally Immunized and Control Eyes}

No difference could be detected in the cell infection rate with strain 5503 between the 14-days cell cultures from the cornea 5 weeks after infection with strain 5503 and those from the control cornea of the other eye (Fig. 3A). Taking account of the dilution of cellular resistance factors due to cell division, younger cell cultures (4-days culture)

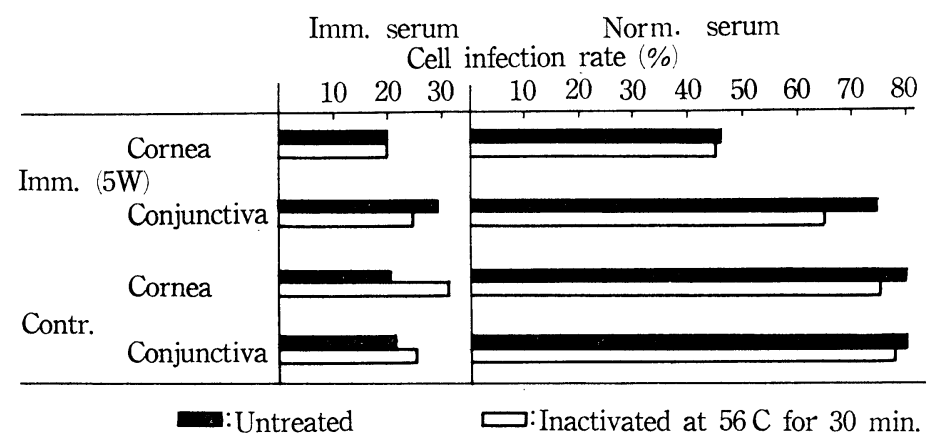

Fig. 4. Effect of hyperimmune serum on infection rates of cell cultures from locally immunized and control eyes challenged with Shigella flexneri 2a strain 5503. 
from the cornea 3 weeks after infection were tested (Fig. 3B). There was no difference either between the cells of pretreated and control eyes.

In the presence of hyperimmune serum, cell infection rates decreased to an equally small extent in the 14-days cultures both from the pretreated eyes and from the control eyes probably due to agglutination of extracellular bacilli (Fig. 4). No significant difference could be found in the cell infection rate or in the growth pattern of intracellular bacilli (Fig. $7 \& 8$ ) between locally pretreated and control groups either in the presence or absence of hyperimmune serum.

\section{Discussion}

In the in vitro experiments for cell infection with the Shigella organisms, such established cell lines as HeLa cells and Henle's intestinal cells were frequently used (Watkins, 1960, Gerber and Watkins, 1961; LaBrec et al., 1964; Ogawa et al., 1967c). Cell infection rates in these cell lines, however, were extremely low, unless the infection medium contained inactivated normal horse serum (Gerber et al., 1961). Furthermore our experiences have shown that the effect of horse serum to promote invasion of the bacilli into cells is fairly variable (unpublished data). For the studies on the pathogenicity of Shigella, which are being approached from the aspects of cell penetration and intracellular growth of the bacilli, therefore, these established cell lines proved unsatisfactory because of low infection rates, and an attempt was made to find cell culture systems with constantly higher infection rates. The results of the present studies gave an infection rate of $10 \%$ or more in the case of the cornea cells of guinea pigs and the lung cells of mice when $S$. flexneri and $S$. dysenteriae 1 were used, while in the case of the primary or the secondary cultures isolated from various embryonic or resected tissues and two stable lines of human origin and the primary or secondary cultures from embryonic tissues of monkeys the infection rates were clearly low. It follows that guinea pig cornea cells and mouse lung cells are most susceptible to Shigella infection, and suitable for the quantitative experiments mentioned above. The question left unsolved yet is whether or not the alteration of infection rates occurs during the subculture period after isolation. It was observed that in the guinea pig cornea cells the infection rate of younger cell cultures was higher than that in cells subcultured 20 times over 9 months, while in the mouse lung cells it was higher after more than 100 subcultures performed over 3 years than in younger cell cultures; L strain showed a low infection rate with all the types of Shigella except S. flexneri 3a strain 23-65. Further study is needed to solve the problem of the correlation between the establishment of cultured cells and the susceptibility to Shigella infection.

In man and monkeys the lesions produced in the case of bacillary dysentery are found principally in the large bowel, and even in guinea pigs and mice the intestinal mucosa are involved in experimental shigellosis. With respect to natural susceptibility to Shigella infection, naturally occurring disease is limited almost to man and monkeys the latter cases being occasional. Dysenteric lesions can also be produced experimentally in the intestinal mucosa of guinea pigs (Formal et al., 1958) and mice (Cooper, 1959) by oral introduction of virulent Shigella organisms under certain conditions. These facts would suggest that natural susceptibility of the animal species to Shigella infection may be in the order of man, the monkey, the guinea pig and the mouse. Hence it would be reasonable to infer that the cell cultures isolated from susceptible animal tissues may give a higher infection rate with Shigella than those from resistant ones. In the present. 
studies this assumption was tested by infecting cell cultures derived from various mammalian tissues with the Shigella organisms. The results indicated that no consistent correlationship between animal species- or organ-specificity with respect to natural susceptibility to Shigella infection and the infection rate in the cell cultures which originated from the corresponding animals or organs. The fact that guinea pig cornea cells were highly susceptible to the infection was expectional, but not that the cells from human or monkey intestine showed low infection rates with all the types of the dysentery bacilli tested. There may be many unknown factors which affect susceptibility of whole animals to Shigella infection in vivo, even if consideration is limited to the cell level. On the other hand, it is doubtful to what degree the isolated cell cultures in vitro, which were controlled in an artificial environment quite different from that in animal bodies, retained physiological characteristics in vivo. As long as experiments depend upon the present cell culture procedure, it might be more reasonable to assume the present findings as an indication the interaction between host cells and bacilli may be independent from that in intact animal bodies.

It is difficult to compare the cell infectivity among types of Shigella tested, since it varies greatly according to the period and the method of preservation of the bacilli (to be published). Only $S$. sonnei constantly showed a low cell infectivity as compared with other types of Shigella, not only in HeLa cells but also in mouse lung cells and guinea pig cornea cells. This finding seems to be related to the following facts. 1) The recent increase in incidences to $S$. sonnei among human bacillary dysentery in Japan has been accompanied by milder symptoms particularly in adults in contrast with the former predominance of those due to $S$. flexneri. 2) Severer symptoms produced by $S$. flexneri than those produced by $S$. sonnei, as well as a greater capability of the former bacilli to penetrate and multiply in epithelial cells of the mucous membrane, were observed in experimental Shigellosis in monkeys and in guinea pigs (to be published).

The experiment on the immunity to Shigella infection using the eyes of guinea pigs (Ogawa et al., 1966b) showed that there was a remarkable increase in resistance in the eyes recovering from keratoconjunctivitis; the fluorescent antibody technique proved that penetration and growth of the challenge bacilli in epithelial cells were kept in check (to be sublished). In the present studies, however, in the absence of hyperimmune serum in infection medium no difference in the cell infection rate was observed between primary cultures of the cornea as well as the conjunctiva of the eye which showed acquired resistance in whole animal experiment and those of the remaining susceptible eye. In the precence of hyperimmune serum the infection rate decreased equally in the eyes recovering from keratoconjunctivitis and in the control eye. This decrease is considered as resulting from agglutination of extracellular bacilli-a mechanical factor. In the intracellular growth pattern of the bacilli, the immunological effect was not proved either, as shown in Fig. $7 \& 8$. To summarize, the factors of the acquired local resistance in the whole animal could not be proved in the primary cell culture in vitro which consisted mainly of polygonal cells in morphology. It will be further necessary to examine the role of the mesenchymal cells as cellular factors in the local resistance, as well as humoral factors. 


\section{ACKNOWLEDGEMENT}

The authors are indebted to Dr. Yasuyuki Egashira, for his encouragement and advice in preparation of this pager.

Thanks are also due to Dr. Hiromichi Mizutani for supplying the human embryo, to Dr. Shigeo Honjo for the monkey embryo and to Dr. Shoshichi Takeuchi for the resected human tissues.

\section{REFERENCES}

Cooper, G. N. (1959): Experimental shigellosis in mice. I. Chronic infection with Shigella dysenteriae type 2. Australian J. Exptl. Biol. Med. Sci., 37, 193-200.

Formal, S. B., DAMin, G. J., LABREC, E. H. AND SchneIder, H. (1958) : Experimental shigella infections: Characteristics of a fatal infection in guinea pigs. J. Bacteriol., 75, 604-610.

Gerber, D. F. \& WATkins, H. M. S. (1961): Growth of shigellae in monolayer tissue cultures. J. Bacteriol., 82, 815-822.

HENLE, G. AND DEINHARDT, F. (1957): The establishment of strains of human cells in tissue culture, J. Immunol., 79, 54-59.

Honjo, S., Takasaka, M., Fujiwara, T., Ando, M., Nakagawa, M., Ogawa, H., TakaHASHI, R. AND IMAIZUMI, K. (1964): Shigellosis in cynomolgus monkeys (Macaca irus). II. Experimental infection with Shigella flexneri $2 \mathrm{a}$ - with special references to clinical and bacteriological findings. Japan. J. Med. Sci. Biol., 17, 307-319.

LaBrec, E. H., Schneider, H., MAgnani, T. J. AND Formal, S. B. (1964) : Epithelial cell penetration as an essential step in the pathogenesis of bacillary dysentery, J. Bacteriol., 88, 1503-1518.

Ogawa, H., Honjo, S., Takasaka, M., Fujiwara, T. ANd Imaizumi, K. (1966a): Shigellosis in cynomolgus monkeys (Macaca irus). IV. Bacteriological and histopathological observations on the earlier stage of experimental infection with Shigella flexneri 2a, Japan. J. Med. Sci. Biol., 19, 23-32.

Ogawa, H., NAKamuRA, A. AND NAKAYA, R. (1966 b) : Immunity to experimental keratoconjunctivitis shigellosa in guinea pigs, Japan. J. Med. Sci. Biol., 19, 209-214.

Ogawa, H., Nakamura, A., Nakaya, R., Mise, K., Honjo, S., Takasaka, M., Fujiwara, T. AND IMAizumi, K. (1967): Virulence and epithelial cell invasiveness of dysentery bacilli, Japan. J. Med. Sci. Biol., 20, 281-294.

WATkins, H. M. S. (1960): Some attributes of virulence in Shigella, Ann. N. Y. Acad. Sci., 88, 1167-1186.

Yoshikura, H., Hirokawa, Y. AND YAMAdA, M. (1966): Growth acceleration of a mouse cell line after infection with Friend leukemia virus, Japan. J. Med. Sci. Biol., 19, 229-238. 


\section{EXPLANATION OF FIGURES}

FIG. 5: The 5th subculture of guinea pig cornea cells $6 \mathrm{hr}$ after infection with $S$. flexneri 2a strain 5503. Polygonal cells susceptible to Shigella infection were subsequently subcultured. Orig. mag. $\times 400$

FIG. 6: Mouse lung cells (established cell line) $6 \mathrm{hr}$ after infection with $S$. flexneri 2a strain 5503. Orig. mag. $\times 400$

FIG. 7: Primary culture from normal (untreated) guinea pig cornea $6 \mathrm{hr}$ after infection with $S$. flexneri 2a strain 5503. Two types of cells were morphologically differentiated: One type (upper half) was polygonal but poorly connected between cells and heavily infected, the other (lower half) was epithelial type and scarcely infected. Orig. mag. $\times 400$

FIG. 8: Primary culture (polygonal type) from guinea pig cornea recovering from keratoconjunctivitis shigellosa $6 \mathrm{hr}$ after challenge with $S$. fexneri 2a strain 5503 . The pattern of intracellular growth of bacilli was the same as that in primary cornea cells from untreated guinea pigs (Fig. 6). Orgig. mag. $\times 400$ 


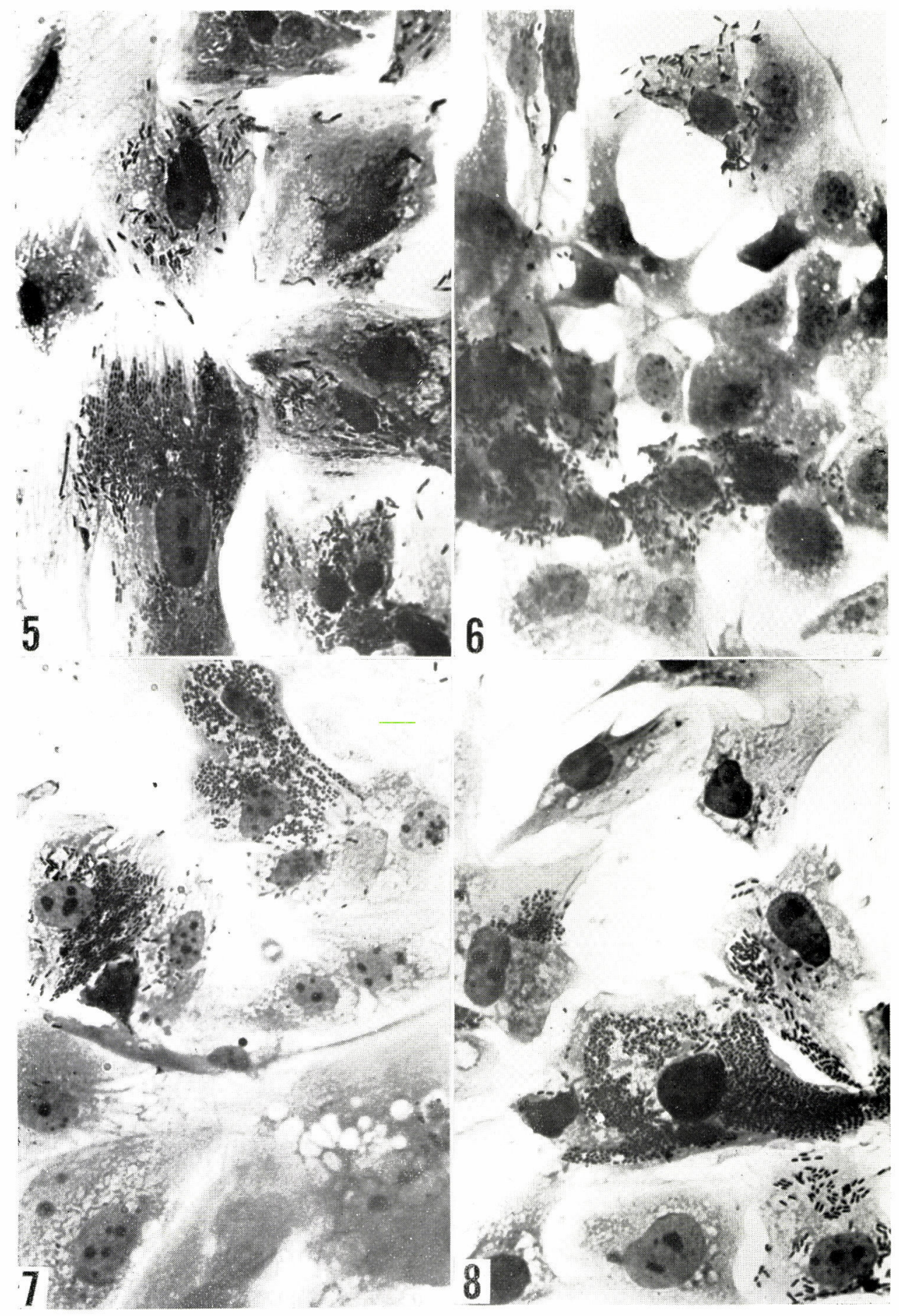

